Archived version from NCDOCKS Institutional Repository http://libres.uncg.edu/ir/asu/

\title{
Appalachỉan
}

B O O N E, N O R T H C A R O L I N A

\section{Sleep Restriction And Circadian Effects On Social Decisions}

\author{
By: David L. Dickinson and Todd McElroy
}

\begin{abstract}
Our study examines how chronic sleep restriction and suboptimal times-of-day affect decisions in a classic set of social tasks. We experimentally manipulate and objectively measured sleep in 184 young-adult subjects, who were also randomly assigned an early morning or late evening experiment session during which decision tasks were administered. Sleep restriction and suboptimal time-of-day are both estimated to either directly or indirectly (via an impact on sleepiness) reduce altruism, trust, and trustworthiness. We conclude that commonly experienced adverse sleep states, most notably chronic sleep restriction, significantly reduce prosocial behaviors, and can therefore limit benefits from short-term social interactions.
\end{abstract}

Dickinson, D. and McElroy, T. (2017). Sleep restriction and circadian effects on social decisions, European Economic Review. Volume 97, August 2017. Pages 57-71. Publisher version of record available at: https://doi.org/10.1016/i.euroecorev.2017.05.002 


\title{
Sleep restriction and circadian effects on social decisions
}

\author{
David L. Dickinson ${ }^{\mathrm{a}, \mathrm{b}, \mathrm{c}, *}$, Todd McElroy $^{\mathrm{d}}$

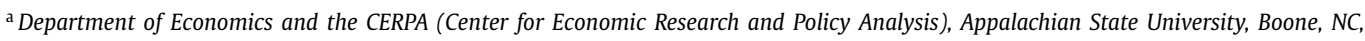 \\ USA \\ ${ }^{\mathrm{b}}$ IZA (Institute for the Study of Labor), Bonn, Germany \\ ${ }^{\mathrm{c}}$ ESI (Economic Science Institute), Chapman University, Orange, CA, USA \\ ${ }^{\mathrm{d}}$ Department of Psychology, Florida Gulf Coast University, Ft. Myers, FL, USA
}

\begin{abstract}
A B S T R A C T
Our study examines how chronic sleep restriction and suboptimal times-of-day affect decisions in a classic set of social tasks. We experimentally manipulate and objectively measured sleep in 184 young-adult subjects, who were also randomly assigned an early morning or late evening experiment session during which decision tasks were administered. Sleep restriction and suboptimal time-of-day are both estimated to either directly or indirectly (via an impact on sleepiness) reduce altruism, trust, and trustworthiness. We conclude that commonly experienced adverse sleep states, most notably chronic sleep restriction, significantly reduce prosocial behaviors, and can therefore limit benefits from shortterm social interactions.
\end{abstract}

Keywords:

Trust

Dictator

Social behavior

Sleep restriction

Circadian

\section{Introduction}

Prosocial behaviors help encourage positive interactions and promote economic institutions that require trust/trustworthiness. There are numerous factors that may influence the propensity to exhibit prosocial behaviors. The variable considered in this paper is one that receives little attention in this area of decision-making research: sleepiness. We examine the impact of commonly experienced sleep and circadian states on outcomes in three well-known simple social interaction tasks: the ultimatum, dictator, and trust games. Prosocial behaviors in these games, such as trust, are at least a simple indication of social capital of the decision maker (Putnam, 1993). Some researchers have even found that increases in survey-based measures of country-level trust promote desirable macroeconomic outcomes, such as increased economic growth (Knack and Keefer, 2007) or reduced government corruption (LaPorta et al., 1997). As such, factors that influence the micro-level choice to behave prosocially-a type of individual investment in social capital (Glaeser et al., 2002)-have multiplier effects in society and the economy, and so the importance of this decision domain should not be underestimated.

The limited research that exists on sleep loss and simple social decisions has utilized highly controlled total sleep loss protocols (Anderson and Dickinson, 2010; Ferrara et al., 2015). Such protocols help establish the dose-response of behavior

\footnotetext{
* Corresponding author at: Department of Economics and the CERPA (Center for Economic Research and Policy Analysis), Appalachian State University, Boone, NC, USA.

E-mail address: dickinsondl@appstate.edu (D.L. Dickinson).
} 
to extreme levels of sleep loss, but the external validity of such findings remains unclear. Observation or field data on sleep levels and choice are highly externally valid, but the limited or lack of experimental control in such data (or the potential bias in self-reports of one's sleep level) reduce one's ability to identify causal effects. In short, we believe that generating primary experimental data using an ecologically valid sleep setting is a valuable approach for studying this particular research question. Here, we utilized an at-home sleep manipulation protocol with objective sleep data acquisition, random treatment assignments, and within-subjects behavioral measures, which increase our ability to claim causal effects. Sleepiness is one of the more concerning health trends at present, and so sleepy decision-making is hardly a rare occurrence. Nevertheless, the weight of the research on sleep and decision making has focused on individual decision tasks (e.g., Harrison and Horne, 2000; Killgore et al., 2006), which leaves a gap in our understanding of how adverse sleep states affect choices in a critical decision domain.

A main contribution of our work is to experimentally manipulate sleep in ecologically valid ways that are highly applicable to the real world-the levels of sleep restriction we examine are pervasive in modern society and part of everyday life for nearly 30\% of U.S. adults (Schoenborn and Adams, 2010). And, while the U.S. Centers for Disease Control and Prevention has labeled sleep deprivation a public health epidemic, little is known about how commonly experienced adverse sleep states impact social interactions. We hypothesize that increased sleepiness will reduce prosocial behaviors given the (limited) related research and given our understanding of deliberative thinking and social decisions (Anderson and Dickinson, 2010; Ferrara et al., 2015; Rilling and Sanfey, 2011; Krajbich et al., 2009; McCabe et al., 2001; Chee and Chuah, 2008). This hypothesis suggests that sleepiness leads to inefficiencies or unrealized benefits in social exchanges.

Results from our 3-week at-home sleep protocol study indicate that both sleep restriction and suboptimal time of day either directly or indirectly (through sleepiness) reduce simple behavioral measures of prosocial decisions. Estimated reductions in dictator giving are the most robust, though our multivariate estimation results show significant decreases for trust and trustworthiness in most specifications as well. Given that these simple games can be considered building blocks for more complex social interactions, such findings have important implications. A significant portion of adults in many countries have habitual sleep levels similar to those we study, and sleep restricted decision-making at suboptimal times of day is common in modern society as well. Our data draw attention to a typically overlooked behavioral "cost" of these modern sleep trends. At least in the area of social interactions, sleepiness may contribute to a type of dead-weight loss of potential benefits that has not been previously highlighted.

\subsection{Background}

Our behavioral hypothesis stems from the argument that prosocial behavior requires deliberative thinking and active suppression of myopic self-interest (Rilling and Sanfey, 2011; McCabe et al., 2001; Fehr and Camerer, 2007; Achtziger et al., 2016). Interestingly, recent evidence suggests that reduced deliberation may promote altruism and cooperation in certain contexts (Rand et al., 2012; Rand et al., 2014; Rand et al., 2016), although other studies found no such relationship (Verkoeijen and Bouwmeester, 2014; Tinghög et al., 2013; Krajbich et al., 2015). It is important to note, however, that these studies typically employ standard cognitive load or time pressure manipulations that are not directly comparable to our manipulation. ${ }^{1}$ Another distinct manipulation in the literature that is intended to reduce deliberation is referred to as "ego depletion", which seems to impact motivational aspects of task performance more than cognition (Inzlicht and Schmeichel, 2012). ${ }^{2}$ Notably, Ainsworth et al. (2014) reported reduced trust following ego depletion, and a study by Vohs et al. (2010) crossed ego-depletion with sleep deprivation and found that only ego-depletion influenced the expression of the negative social behavior of aggression. Thus, the existing research leads to the natural question of whether we believe our sleep manipulations will affect pro-social behavior by harming cognition or task motivation.

It has been argued that sleep loss may impact motivation as well as cognition, but the mixed evidence includes examples where no significant motivation decline is reported (e.g., Drummond et al., 2004), while others report significant motivational decline following sleep deprivation (e.g., Almklov et al., 2015). However, even when short, novel, or incentivized tasks are used to mitigate motivational decline caused by sleep loss (see Alhola and Polo-Kantola, 2007, and sources therein), performance decrements are still observed. This argues that cognitive effects likely dominate any motivation effects of our treatment manipulation-our tasks are incentivized, short, and relatively novel for the subjects. Another recent study concludes that self-regulation (the target of ego depletion manipulations) and fatigue are not overlapping constructs (Vohs et al., 2010), which supports our view of dominant cognition effects over motivational effects in our design. Thus, compared to commonly used alternative protocols aimed at reducing deliberative processes, ours is unique and focused on cognitive effects of mild adverse sleep states. We also argue that our ecologically valid at-home sleep protocol implies that our results have more clear implications for real world decision makers.

For our research question, another relevant stream of literature has identified the importance of deliberative thinking for prosocial decisions (Rilling and Sanfey, 2011; Krajbich et al., 2009; McCabe et al., 2001; Fehr and Camerer, 2007). This is important because the prefrontal cortex (PFC) is particularly vulnerable to sleep deprivation. Some studies report negative sleep impacts on PFC function (Horne, 1993; Muzur et al., 2002; Chee and Chua, 2008), and Horne (1993) reports a

\footnotetext{
${ }^{1}$ Distinct from the other studies mentioned, Krahbich et al. (2015) examined patients with prefrontal lesions.

${ }^{2}$ With an ego depletion manipulation, a subject is made to exert self-control of some sort at time $t$, and then administration of another task requiring self-control is administered at time $t+1$. Ego depletion is meant to reduce one's capacity to self-regulate in the time $t+1$ task.
} 
more general link between sleep deprivation and reduced PFC activation. This is consistent with recent neural and behavioral studies showing the negative impact of adverse sleep or circadian states on deliberative decisions (Yoo et al., 2007; McElroy and Dickinson, 2010; Dickinson and McElroy, 2010). However, in other contexts researchers have reported increased PFC activation following sleep deprivation. For example, compensatory PFC activation on a verbal learning task is reported in Drummond et al. (2000) following total sleep loss. Regarding decision making studies, Venkatraman et al. (2011) documented increased ventromedial PFC (vmPFC) activation following sleep deprivation in a risk-taking task. The authors attributed the increase in vmPFC activation following sleep loss to an increased focus on monetary gains. However, rather than improving decision making, increased vmPFC activation appears to suggest an optimism bias triggered by sleep loss (see also Venkatraman et al., 2007, 2009). Our interpretation of this literature is that sleep deprivation, while increasing some PFC activation on certain tasks, has not been found to improve deliberative processes useful for optimal decision making. In fact, others have noted the negative impact of sleep loss on decisions that rely particularly on the vmPFC (Killgore et al., 2012).

Somewhat related to the issue of PFC importance in social decisions, researchers have reported higher altruism and trust levels in older adolescents (Benenson et al., 2007; Fehr et al., 2008; Fehr et al., 2013). Such findings also support the hypothesis that the PFC, which develops later than other brain regions, is important for prosocial choice. As a whole, we find that the weight of the existing evidence suggests PFC vulnerability to adverse sleep or circadian states, and PFC function is important for prosocial choice. While this is a somewhat overly simplified characterization of the literature, it is useful in organizing our thinking and forming our hypothesis regarding the likely impact of our experiment manipulation in the domain of social choice. Our hypothesis can be succinctly stated as follows:

Hypothesis: Sleepy subjects-whether from sleep restriction or suboptimal time of day-will make less prosocial decisions.

\subsection{Experimental protocol}

Our starting point was to first generate a database of young-adults (18-39 year olds) for whom we had specific sleeprelated information-some of this was to address inclusion criteria for the main experimental study and some was in order to manage random session-time assignments. We first administered a large-scale online survey that included questions on basic demographics, recent sleep habits, validated anxiety and depressive disorder screener questions, and a validated morningness-eveningness questionnaire. Over multiple waves of the online survey we generated several thousand responses to our survey (mostly student responses). ${ }^{3}$ Criteria for viable subjects for the main study included: those below standard cutoffs for risk of major depressive or anxiety disorder, no diagnosed sleep disorder or self-reported insomnia, between 18 and 39 years of age. To assess diurnal preference, the survey administered a validated short form of the morningnesseveningness questionnaire, henceforth rMEQ (Adan and Almiral, 1991). The rMEQ classifies individuals on a continuous scale of 4-25, with morning-types having a rMEQ score from 18 to 25 and evening-types having a rMEQ score from 4 to 11 . Though based on subject self-reports, the methodology has been validated against physiological data on oral temperatures (Horne and Östberg, 1976) and is a standard tool in circadian research. A validated measure of diurnal preference was necessary for the validity of our random session time assignments.

We identified morning-types (MT) and evening-types (ET) in the database and randomly assigned each potential subject, ex ante, to a morning (7:30 a.m.) or an evening (10:00 p.m.) experiment session prior to sending out recruitment emails. Due to the rarity of true MT subjects-less than $10 \%$ in young adult populations are morning-types (see Chelminski et al., 2000)-we extended our rMEQ cutoff for MT to include rMEQ scores of 16 and 17. To compensate, we only recruited the more extreme (and still abundant) ET subjects with rMEQ scores from 4 to $9 .{ }^{4}$ This approach helped ensure that the sample we recruited for the main study was comprised of individuals with true morningness or eveningness preferences. ${ }^{5}$

For the main study, subjects were recruited for a 3-week experiment protocol with multiple in-lab sessions each occurring at the same randomly assigned time-of-day for that subject. The 3-week protocol allowed for a within-subjects manipulation of at-home sleep levels, as discussed in the next paragraph. Another design option would have been to vary the time of day for the lab sessions such that each subject was administered decision tasks both at a more optimal (circadian matched) and less optimal (circadian mismatched, MM) time of day. That is, a within-subjects circadian mismatch protocol was an alternative design choice. However, one of our concerns was subject scheduling and the difficulties in recruiting and preserving participation of subjects with non-constant session times-of-day, and so we chose to proceed with the circadian portion of the protocol as a between-subjects design feature (see Dickinson et al., 2017, for a more complete discussion of subject attrition in this design). Other researchers may wish to examine optimal versus sub-optimal times-of-day in decision

\footnotetext{
${ }^{3}$ While some have argued that laboratory experiments may overstate social behaviors (Levitt and List, 2007), recent research suggests that the use of student subject pools may, if anything, underestimate the importance of social behaviors in the general population (Falk et al., 2013). What is important is to note that the qualitative direction of our results suggest damaging effects of relatively mild adverse sleep states on prosocial behaviors.

${ }^{4}$ In this way, our sample was still drawn from the tails of the rMEQ distribution and eliminated the same amount of support from the non-tail portion of the rMEQ distribution compared to if we had used the traditional MT cutoff $(\mathrm{rMEQ}=18)$ but included non-extreme $\mathrm{ET}(\mathrm{rMEQ}=10-11)$ in our sample.

${ }^{5}$ Others studies have assessed diurnal preferences only after recruitment, and then used a median split to classify subjects as morning-types or evening-types (e.g., Bodenhausen, 1990; Kruglanski and Pierro, 2008). Given the distribution of diurnal preferences among young adult subjects (see Chelminski et al, 2000), the approach will incorrectly classify many intermediate or indeterminate type subjects as morning-types.
} 
SR treatment week $=$ prescribed 5-6 hr/night in bed attempting to sleep, naps discouraged. Sleep diaries kept.

WR treatment week = prescribed 8-9 $\mathrm{hr} /$ night in bed attempting to sleep, naps discouraged. Sleep diaries kept.

Ad lib sleep week $=$ subject sleep however much/little they like. Sleep diaries kept.

Sessions: Subjects come to research lab in each instance.

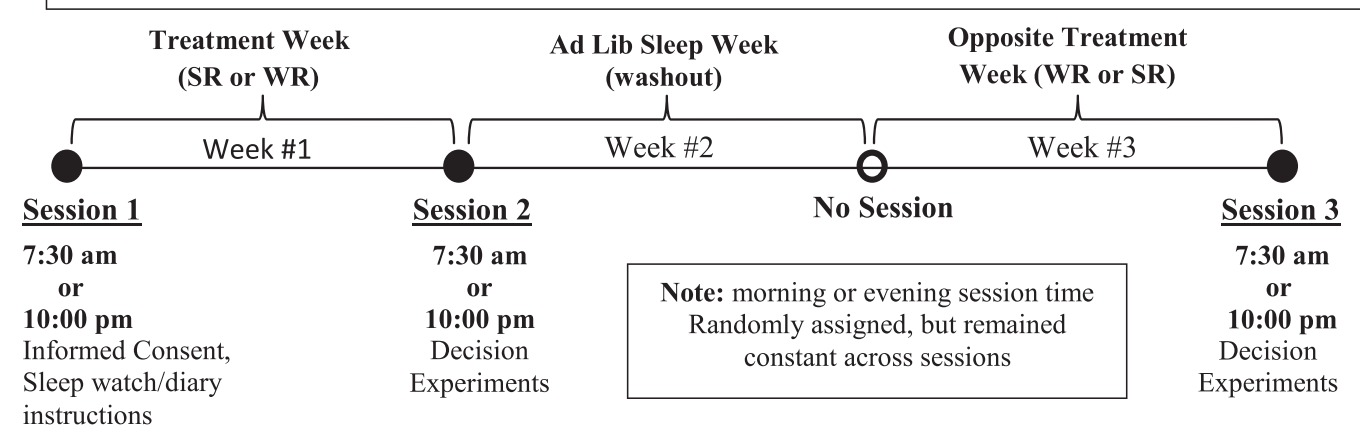

Fig. 1. Protocol details and timeline.

Note: Figure reproduced from Dickinson et al. (2017).

making as a within-subject design factor, but our design choice was to favor additional statistical power along the at-home sleep restriction dimension.

Subjects were recruited in groups (average size was about 13 subjects) and, while each experimental group was either designated a "morning session" or "evening session" group, there was a mix of morning-type and evening-type subjects in each group. Fig. 1 shows the timeline of the protocol and when the decision experiment sessions took place. As can be seen, each subject was prescribed a well-rested (WR: 8-9 h/night in bed) and a sleep-restricted (SR: $5-6 \mathrm{~h} / \mathrm{night}$ in bed) week with an ad-lib sleep week in between. A small number $(n=30)$ of control subjects were recruited as well to assess pure repeat administration effects. Control subjects were intermediate diurnal preference individuals, attended experiment sessions at mid-day (neither early nor late), and they were assigned WR sleep weeks in both Week 1 and Week 3 of the protocol.

The set of bargaining tasks was administered at the end of weeks 1 and 3 (i.e., at the end of each sleep treatment week). Because the session time-of-day remains constant for a given subject, this is a mixed design with between-subjects (circadian match/mismatch) and within-subjects (SR/WR) components. Subject sleep levels are objectively monitored during the entire 3 weeks with actigraphy devices that allow for raw data output (see Appendix for model of devices, which are commonly used in sleep and clinical research and are well-validated). Scoring the sleep data involved complementary sleep diary input as well as the objective actigraphy data, and subjects were paid a separate fixed payment (\$80) for providing the 3 weeks of sleep data. Subjects were deemed compliant if average nightly sleep during the WR week was at least 60 min more than during the SR week. The Appendix discusses the legitimacy and data-driven approach to this standard for compliance in detail (see also Dickinson et al., 2017, for more extensive details and analysis of the sleep protocol).

\subsection{Decision tasks}

We administered the ultimatum (Güth et al., 1982), Dictator game (Forsythe et al., 1994), and Trust games (Berg et al., 1995) twice for each subject-once following SR and once following WR. For the Ultimatum and Dictator games we used a $\$ 10$ starting sum. In the Ultimatum game, the first-mover proposes a division of the $\$ 10$ and the second-mover may either accept or reject the offer. Both players receive a zero payoff if the offer is rejected. The Dictator game removes the secondmover decision from the game, such that the dictator simply decides how the $\$ 10$ will be divided between the two players. For the $\$ 10$ Trust game we did not endow the responder with any money, (and so, a subject attempting to equalize firstmover/second-mover earnings will send back a different amount than if both first and second-mover were endowed with $\$ 10$ ). The first mover may choose to pass (trust) some, all, or none of the $\$ 10$ to the second-mover. Whatever is trusted to the second mover is then tripled by the experimenter, and the second-mover can then choose to pass back some, all, or none of the tripled amount. The amount passed back is generally viewed as a measure of trustworthiness. We also administered a "risk" version of the Trust game where the second-mover is a pass-back algorithm rather than another subject in the group. In this way, we can identify the importance of the social interaction, as opposed to the pure risk of passing money, in making the interpersonal decision (see Sanfey et al., 2003; Kosfeld et al., 2005).

The games were administered via the strategy method whereby all subjects made decisions as first-mover and secondmover prior to knowing to which role he/she was randomly assigned. This procedure requires subjects to make decisions as a second-mover in the Ultimatum and Trust games for all possible contingencies of what the first mover might do. It was common knowledge that we would make random role assignments (first- or second-mover), anonymous counterpart assignments, and randomly select one of games for payoff for that decision session only after all decisions were made for all tasks. Outcomes for the randomly selected payoff game were only revealed at the end of the decision session, when 
Table 1

Sample size per design cell (treatment subjects).

\begin{tabular}{|c|c|c|}
\hline & Morning session & Evening session \\
\hline \multirow{3}{*}{$\begin{array}{l}\text { Morning-type } \\
\text { Evening-type }\end{array}$} & $34(30)$ & $38(28)$ \\
\hline & $39(30)$ & $38(31)$ \\
\hline & \multicolumn{2}{|c|}{$\begin{array}{c}\text { Sample size }=149 \text { subjects } \\
\text { Matched obs }=76, \text { Mismatched obs }=73 \\
\text { (compliant and sleep data intact shown in parenthesis) }\end{array}$} \\
\hline
\end{tabular}

Notes: Bold values indicate circadian mismatches.

Table reproduced from Dickinson et al. (2017).

payoffs for these and other decision tasks were given out in cash. ${ }^{6}$ During the second decision session (session 3 in Fig. 1 ) subjects were informed that the roles, counterparts, and the payoff game were re-randomized (i.e., not the same as in the first decision session).

In total, we recruited 184 young adults (30 control: 154 treatment: $18-39$ years old, mean $21.66 \pm 4.43$ years old) to participate in the 3-week study. The relevance of our results to real world decision makers are, in large part, due to an at-home sleep and circadian protocol with high ecological validity. Table 1 shows the mix or circadian matched versus mismatched subjects for the between-subjects component of the design. Of these 184 subjects, 149 (30 control: 119 treatment) were deemed compliant based on objective actigraphy data. ${ }^{7}$ Compliant treatment subjects slept a minimum of $1 \mathrm{~h}$ more per night during the SR week than the WR week, but there was variation in the level of compliance (e.g., the average difference between WR and SR nightly sleep was about $1.5 \mathrm{~h}$ ). The results below are based on the subset of compliant treatment subjects $(n=119)$.

\section{Results}

Subjects self-reported sleepiness during the decision sessions using the Karolinska Sleepiness scale (Åkerstedt and Billberg, 1990; Kaida et al., 2006). To test treatment manipulations we regress self-report sleepiness on treatment, demographics, and sleep control variables. Both SR and circadian mismatch significantly increased sleepiness $(p<.01)$, as noted below, ${ }^{8}$ which is evidence of the validity of the protocol at manipulating sleepiness. In Table 2 we present descriptive statistics of the behavioral outcomes from each task, along with nonparametric tests of means. Nonparametric tests of the SR treatment effect uses the signed-rank test for matched data given each subject makes decisions under both $S R=0$ and $S R=1$ conditions. For testing means of behavioral outcomes resulting from circadian mismatch (MM), we used the Mann-Whitney test for unmatched data after first averaging each subject's decisions for a task across the two decision sessions. Given our experimental design choice, the test for MM effects is inherently less statistically powerful than our tests of SR effects on behavioral outcomes. Results in Table 2 indicate marginally significant SR effects on Dictator offers and trust decisions. Of course, such tests do not take into account other covariates, and so a more controlled statistical analysis follows.

We next present results from the multivariate analysis. The general model structure we estimate is: $Y_{t}=a+\mathbf{X} \boldsymbol{\beta}+\mathbf{Z} \boldsymbol{\gamma}$ where $Y_{t}$ is the behavioral outcome, $Y$, for task, $t$. $\mathbf{X}$ represents a vector of sleep-related variables, which includes the experimentally manipulated measures of sleep restriction and circadian mismatch and a measure of chronic daytime sleepiness, Epworth sleepiness, which is derived from the pre-screen sleep survey. Note that Epworth sleepiness is a separate construct from state-level Karolinska self-reported sleepiness elicited during the decision sessions (simple correlation $\rho=.12$ between these two measures). $\mathbf{Z}$ includes indicators for the session number (Session 3 dummy indicates the second administration of the task) and timing (Morning Session=1), as well as demographic controls such as age, gender, and sub-clinical depression and anxiety risk scores. ${ }^{9}$ We estimated the models using a random effects error structure for multiple observations per subjects.

Estimation results in Tables 3-6 identify predictors of the key outcome variables for each social decision task. The relevant treatment variables for sleep restriction and circadian mismatch are highlighted. The treatment variable, Mismatch, is an indicator variable equal to 1 if the subject is mismatched relative to her more preferred time of day. Because there exists variation in the level of compliance among subjects deemed compliant, a continuous measure of sleep level is of interest.

\footnotetext{
${ }^{6}$ Note that the fixed payment for participation was separate from the cash incentive payments for outcomes in the decision experiments. Subjects could receive the $\$ 80$ fixed payment by check or Amazon gift code, which was sent out several days after the end of the experiment so that sleep data could be first checked for instances of apparent gross noncompliance. The compliance standard for issuing experiment payment was more liberal than the standard we used for considering the sleep data compliant with the treatment condition. In general, to maintain satisfaction in the subject pool, our position was to fully pay subjects for what appeared a good faith effort at compliance, even if data were ultimately considered noncompliant for analysis purposes. This more liberal payment policy was not known to subjects during their participation.

7 A small number of treatment-subject observations were lost due to malfunction or corruption of actigraphy devices (9 subjects). The final sample of 119 compliant subjects and 30 control subjects all had complete actigraphy data records.

8 The full results of this estimation are shown in Table 6, which is the first stage regression of the two-stage endogenous variables regression used to evaluate sleepiness as a mediating variable in evaluating behavioral outcomes.

${ }^{9}$ Recall that subjects at clinical risk levels for depressive or anxiety disorder were not recruited for the study.
} 
Table 2

Behavioral summary statistics and nonparametric treatment effect tests (sample is $\mathrm{n}=119$ compliant treatment subjects. Data pooled across sessions and subjects).

\begin{tabular}{|c|c|c|c|c|c|c|}
\hline Variable & Ult $\$ \$$ Offer & Ult $\$ \$$ MAO & Dictator $\$ \$$ Offer & Trust person $\$ \$$ Offer & Trust distribution $\$ \$$ Offer & Trust-worthiness \% passback \\
\hline $\mathrm{SR}=0$ & $4.49(1.04)$ & $2.87(1.77)$ & $3.44(2.00)$ & $5.19(2.47)$ & $4.62(2.58)$ & $.274(.147)$ \\
\hline $\mathrm{SR}=1$ & $4.42(1.01)$ & $2.89(1.82)$ & $3.02(2.24)$ & $4.77(2.27)$ & $4.56(2.49)$ & $.261(.146)$ \\
\hline $\begin{array}{l}\text { Signed-rank } \\
\text { (Matched) }\end{array}$ & $\begin{array}{l}Z=-.57 \\
p=.57\end{array}$ & $\begin{array}{l}Z=-.14 \\
p=.88\end{array}$ & $\begin{array}{l}\mathrm{Z}=-1.80 \\
p=.07\end{array}$ & $\begin{array}{l}Z=-1.65 \\
p=.10\end{array}$ & $\begin{array}{l}Z=-.09 \\
p=.93\end{array}$ & $\begin{array}{l}Z=-.59 \\
p=.56\end{array}$ \\
\hline $\begin{array}{l}\mathrm{MM}=0 \\
\mathrm{MM}=1 \\
\text { Mann-Whitney } \\
\text { (Unmatched) }\end{array}$ & $\begin{array}{l}4.42(1.04) \\
4.49(1.02) \\
Z=-.08 \\
p=.93\end{array}$ & $\begin{array}{l}2.77(1.75) \\
3.00(1.84) \\
Z=-.69 \\
p=.49\end{array}$ & $\begin{array}{l}3.33(2.01) \\
3.13(2.25) \\
Z=.69 \\
p=.49\end{array}$ & $\begin{array}{l}5.04(2.34) \\
4.92(2.43) \\
Z=.93 \\
p=.35\end{array}$ & $\begin{array}{l}4.43(2.42) \\
4.86(2.63) \\
Z=-1.14 \\
p-.25\end{array}$ & $\begin{array}{l}.268(.137) \\
.267(.156) \\
Z=.24 \\
p=.80\end{array}$ \\
\hline
\end{tabular}

Note: Significance levels are for 2-tailed tests. Test on SR treatment effect use matched data, whereas MM effect tests use unmatched data on each subject's average behavioral effect across both decision sessions. Alternative nonparametric tests (Sign test of SR effect; Median test of MM effect) produce similar results. For those SR effects on Dictator Offers and Trust decision, which had $p \leq .10$ for the 2-tailed signed-rank test, the corresponding p-values of the Sign (binomial) test are $p=.13$, and $p=.05$, respectively. 
Table 3

Ultimatum decisions.

Random effects GLS regression (for full sample).

Dependent variable $=$ Portion of $\$ 10$ pie offered to 2nd-mover.

\begin{tabular}{|c|c|c|c|c|}
\hline \multirow[b]{2}{*}{ Variable } & \multicolumn{2}{|c|}{ Ultimatum offers ( $\mathrm{n}=234$ obs $)$} & \multicolumn{2}{|c|}{ Minimum acceptable offer $(\mathrm{n}=236$ obs) } \\
\hline & $\begin{array}{l}\text { (1) } \\
\text { Coef (st. error) }\end{array}$ & $\begin{array}{l}(2) \\
\text { Coef (st. error) }\end{array}$ & $\begin{array}{l}(3) \\
\text { Coef (st. error) }\end{array}$ & $\begin{array}{l}(4) \\
\text { Coef (st. error) }\end{array}$ \\
\hline Constant & $4.70(.60)^{* * *}$ & $5.29(.54)^{* * *}$ & $4.71(1.01)^{* * *}$ & $4.70(.94)^{* * *}$ \\
\hline Female $(=1)$ & $-.29(.18)$ & $-.27(.18)$ & $-.61(.31)^{* *}$ & $-.62(.31)^{* *}$ \\
\hline Age & $-.02(.02)$ & $-.02(.02)$ & $-.08(.04)^{* *}$ & $-.08(.04)^{* *}$ \\
\hline Depression score & $.10(.12)$ & $.11(.12)$ & $.02(.21)$ & $.02(.21)$ \\
\hline Anxiety score & $-.02(.04)$ & $-.02(.04)$ & $.06(.07)$ & $.06(.07)$ \\
\hline Epworth score & $-.01(.03)$ & $-.01(.03)$ & $-.03(.04)$ & $-.03(.04)$ \\
\hline Session \#3 & $-.18(.09)^{* *}$ & $-.18(.09)^{* *}$ & $-.59(.13)^{* * *}$ & $-.59(.13)^{* * *}$ \\
\hline Morning Session $(=1)$ & $.10(.17)$ & $.10(.17)$ & $.47(.30)$ & $.47(.30)$ \\
\hline Morningness score & $.01(.02)$ & $.01(.02)$ & $.02(.03)$ & $.02(.03)$ \\
\hline Circadian Mismatched $(=1)$ & $.02(.17)$ & $-.004(.17)$ & $.11(.30)$ & $.11(.30)$ \\
\hline Nightly Sleep (min) & $.001(.001)$ & - & $-.00003(.001)$ & - \\
\hline Personal SD (min/night) & - & $-.0014(.0007)^{* *}$ & - & $.00001(.001)$ \\
\hline Wald chi-squared test (10) & 9.43 & 12.32 & $31.68^{* * *}$ & $31.75^{* * *}$ \\
\hline
\end{tabular}

Notes: *, **,*** indicate significance at the $.10, .05$, and .01 levels, respectively, for the 2-tailed test.

For each decision outcome, two models are estimated to allow for different approaches to scoring sleep levels: The first model uses average nightly actigraphy-measured sleep during the week prior to the decision, Nightly Sleep, as the continuous variable measure for sleep levels. The second model uses a constructed hybrid measure, Personal Sleep Deprivation, which is the difference between a subject's self-reported optimal nightly sleep (from the earlier online sleep survey) and the actigraphy-measured nightly sleep the week prior to the decision. In a sense, the variable Personal $S D$, handicaps each individual for his/her perceived personal sleep need. Additional estimations are included in the Appendix where sleep restriction is coded as a dichotomous indicator variable $(S R=0,1)$ to identify the SR treatment week. Regarding the non-treatment control variables, the Session 3 indicator is robustly significant across estimations of the different behavioral outcome measures. The sign of the coefficient on Session 3 captures a general tendency to behave in a more self-interested fashion during the last session. ${ }^{10}$

The estimated coefficients on the circadian mismatch and sleep level variables allow for a test of our behavioral hypothesis for each model. The estimated direct effect of circadian mismatch on behavioral outcomes is insignificant across all models (Tables 3-6), and so we turn our attention to the statistically significant $(p \leq .05)$ estimated direct effect of sleep restriction on behavioral outcomes-these are an indication that sleep restriction may have a greater impact on behavior than circadian mismatch, but we should also remind the reader that the circadian mismatch dimension of the protocol has less statistical power than the sleep restriction dimension. Regarding sleep restriction, we find significant and robust effects on decisions in the Dictator and Trust games. Results from the Ultimatum game are less convincing, which should not be surprising. The Ultimatum game is standard in this suite of tasks, but it confounds self-interest with risk of a zero payoff in both first- and second-mover roles (or, the desire to accumulate money is in conflict with the desire for fairness, as noted in Sanfey et al., 2003). We find weak evidence that ultimatum offers may be less generous for higher levels of Personal SD (i.e., the more sleep deprived. See Table 3, Model 3), but this effect is not robust across alternative coding of the sleep restriction variable (see Table 2 and Appendix).

Turning to the Dictator game, Table 4 identifies a robust result of reduced Dictator offers when one is sleep restricted (see also Appendix), which is consistent with our hypothesis. The predicted relationship between Dictator offers and Personal SD is shown graphically in Fig. 2-demographics, session identifier, and sleep related control variables are held constant. Given the lack of a rejection threat in the Dictator game, offers in this game are more easily interpreted as a measure of altruism or prosocial behavior than are decisions in the Ultimatum game results. ${ }^{11}$ The size of the estimated effect is also non-trivial. Compared to a fairly typical $\$ 3$ offer in a Dictator game when not sleep-restricted (Personal $S D=0$ ), that same individual being $2 \mathrm{~h}$ sleep-restricted per night ( $120 \mathrm{~min}$ ) chronically sleep restricted would offer $\$ 2.40$. This represents a $20 \%$ reduction in Dictator giving level. ${ }^{12}$

\footnotetext{
10 The same is generally true of results from the Control subjects, although there are only 30 such subjects. The only generally significant variable is the Session 3, variable which, when statistically significant, indicates and increased greed in choice. Unlike the data from the Treatment subjects, however, Control subjects are not significantly less trustworthy during the last session than the first decision session. Because of the small sample of Control Subjects, and the fact that each Treatment subject serves as his/her own baseline regarding the sleep restriction effect, we do not report Control Subject estimations here. They are available on request.

11 Of course, the Dictator game is not itself without confounds in interpretation as Dictator giving may reflect concerns that the experimenter knows of one's decision, or may reflect aversion to payoff inequality (see Fehr and Schmidt, 1999). Nevertheless, such factors are present in both the SR and WR decisions of subject, and any amount of giving intended to reduce inequality aversion can be considered a different form of prosocial behavior.

12 The magnitude of the estimated effect is only slightly smaller-about a $16 \%-17 \%$ reduction-if using Nightly Sleep (model 1 , Table 4 ) or a dichotomous scoring of SR (see Appendix, Table S2). In general, the magnitude as well as the significance of each estimated effect is similar, if not greater, using these
} 
Table 4

Dictator decisions.

Random effects GLS regression (for full sample).

Dependent variable $=$ Portion of $\$ 10$ pie offered to 2 nd-mover.

\begin{tabular}{lll}
\hline Dictator offers $(\mathrm{n}=237$ obs $)$ & $(1)$ & $(2)$ \\
Variable & Coef (st. error) & Coef (st. error) \\
\hline Constant & $1.39(1.22)^{* * *}$ & $3.96(1.05)^{* * *}$ \\
Female $(=1)$ & $-.03(.36)$ & $.06(.35)$ \\
Age & $-.01(.04)$ & $-.02(.04)$ \\
Depression score & $-.23(.23)$ & $-.18(.23)$ \\
Anxiety score & $.11(.08)$ & $.10(.08)$ \\
Epworth score & $.06(.05)$ & $.06(.05)$ \\
Session \#3 & $-.71(.20)^{* * *}$ & $-.72(.20)^{* * *}$ \\
Morning session $(=1)$ & $.29(.34)$ & $.31(.33)$ \\
Morningness score & $-.01(.03)$ & $-.02(.03)$ \\
Circadian mismatched $(=1)$ & $-.22(.34)$ & $-.32(.33)$ \\
Nightly sleep (min) & $.004(.002)^{* *}$ & - \\
Personal SD (min/night) & - & $-.005(.002)^{* * *}$ \\
Wald chi-squared test $(10)$ & $23.02^{* * *}$ & $29.36^{* * *}$ \\
\hline
\end{tabular}

Notes: ${ }^{*},{ }^{* *},{ }^{* *}$ indicate significance at the $.10, .05$, and .01 levels, respectively, for the 2 -tailed test.

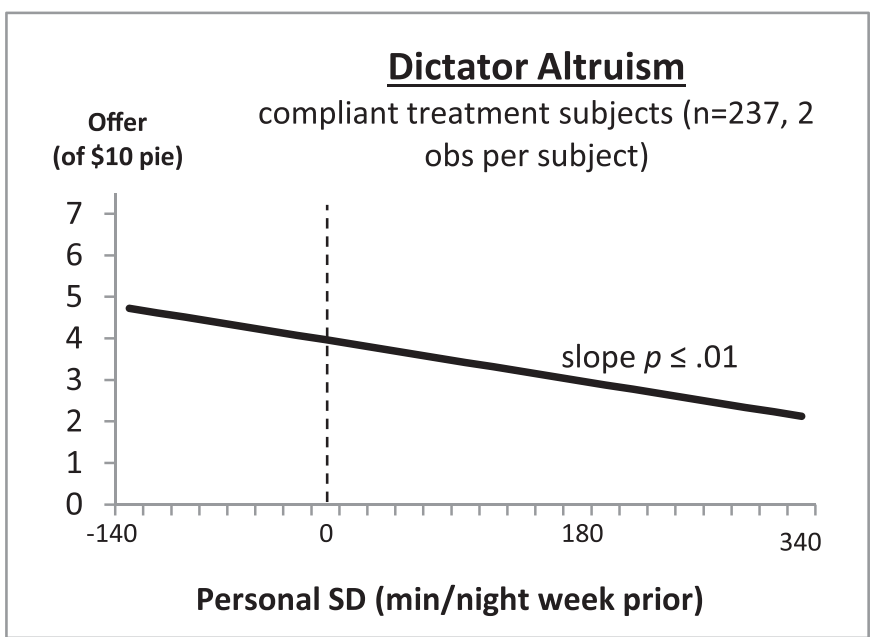

Fig. 2. Predicted dictator offers (altruism)

Notes: Forecast derived from Table 4 with levels of all statistically insignificant variables set to zero. This figure shows range of values of Personal SD observed in sample of compliant treatment subjects. Results show that when dictators are more chronically sleep restricted, they are less altruistic.

Tables 5 and 6 show additional evidence of decreased prosocial behavior in the Trust game when sleep restricted. Table 5 also includes results from a "risk" version of the Trust game where it is common knowledge for subjects that the second-mover is an automated pass-back algorithm. ${ }^{13}$ As can be seen in Table 5, the significant reduction in trust due to sleep restriction occurs only when trust is imbedded in a social interaction (albeit a one-shot and anonymous interaction in this task). The reduction in trust is also significant because it documents how commonplace levels of sleep restriction introduce an inefficiency into the domain of social interactions. The predicted Trust game result is depicted in Fig. 3, and the magnitude of reduced initial trust amount indicates a lost potential in the 2-person exchange that is economically significant. The size of the predicted reduction in trust is similar to the reduction in the Dictator game (\$.50-\$.60), but this represents a slightly smaller effect in percentage terms relative to typical trust levels, which are around $\$ 5-\$ 6$ out of a $\$ 10$ pie (see Berg et al., 1995). 
Table 5

Trust decisions.

Random effects GLS regression (for full sample).

Dependent variable $=$ Portion of $\$ 10$ pie trusted to 2 nd-mover.

\begin{tabular}{|c|c|c|c|c|}
\hline \multirow[b]{2}{*}{ Variable } & \multicolumn{2}{|c|}{ Trust person (Social) ( $\mathrm{n}=230$ obs) } & \multicolumn{2}{|c|}{ Trust algorithm (Asocial) $(\mathrm{n}=236$ obs $)$} \\
\hline & $\begin{array}{l}(1) \\
\text { Coef (st. error) }\end{array}$ & $\begin{array}{l}(2) \\
\text { Coef (st. error) }\end{array}$ & $\begin{array}{l}\text { (3) } \\
\text { Coef (st. error) }\end{array}$ & $\begin{array}{l}(4) \\
\text { Coef (st. error) }\end{array}$ \\
\hline Constant & $3.91(1.39)^{* * *}$ & $6.51(1.18)^{* * *}$ & $4.48(1.48)^{* * *}$ & $5.40(1.28)^{* * *}$ \\
\hline Female $(=1)$ & $-.33(.40)$ & $-.24(.39)$ & $-.47(.43)$ & $-.45(.42)$ \\
\hline Age & $-.01(.04)$ & $-.01(.04)$ & $.01(.05)$ & $.01(.05)$ \\
\hline Depression score & $-.27(.26)$ & $-.23(.26)$ & $-.39(.28)$ & $-.36(.28)$ \\
\hline Anxiety score & $.10(.09)$ & $.09(.09)$ & $.10(.09)$ & $.09(.09)$ \\
\hline Epworth score & $.01(.06)$ & $.01(.06)$ & $-.03(.06)$ & $-.03(.06)$ \\
\hline Session \#3 & $-.64(.25)^{* * *}$ & $-.63(.25)^{* *}$ & $-.02(.25)$ & $-.04(.25)$ \\
\hline Morning session $(=1)$ & $.26(.38)$ & $.27(.38)$ & $-.32(.41)$ & $-.31(.40)$ \\
\hline Morningness score & $-.06(.04)$ & $-.06(.04)$ & $-.02(.04)$ & $-.03(.04)$ \\
\hline Circadian mismatched $(=1)$ & $-.13(.37)$ & $-.21(.37)$ & $.52(.40)$ & $.47(.40)$ \\
\hline Nightly sleep (min) & $.005(.002)^{* *}$ & - & $.001(.002)$ & - \\
\hline Personal SD (min/night) & - & $-.004(.002)^{* *}$ & - & $-.003(.002)$ \\
\hline Wald chi-squared test (10) & 15.44 & $16.07^{*}$ & 6.91 & 8.58 \\
\hline
\end{tabular}

Notes: ${ }^{*},{ }^{* *},{ }^{* *}$ indicate significance at the $.10, .05$, and .01 levels, respectively, for the 2-tailed test

Table 6

Trustworthiness decisions.

Random effects GLS regression (for full sample).

Dependent variable = average \% returned (from strategy choice set).

\begin{tabular}{|c|c|c|c|}
\hline $\begin{array}{l}(\mathrm{n}=234 \text { obs }) \\
\text { Variable }\end{array}$ & $\begin{array}{l}\text { DV = Avg } \\
\text { trustworthiness } \\
\text { over all } \\
\text { amounts } \\
\text { trusted } \\
\text { (1) } \\
\text { Coef (st. error) }\end{array}$ & $\begin{array}{l}\text { DV = Avg } \\
\text { trustworthiness } \\
\text { over all } \\
\text { amounts } \\
\text { trusted } \\
\text { (2) } \\
\text { Coef (st. error) }\end{array}$ & $\begin{array}{l}\text { DV = Avg trustworthiness for amounts } \geq \$ 5 \text { trusted } \\
\text { ( } 3 \text { ) } \\
\text { Coef (st. error) }\end{array}$ \\
\hline Age & $.001(.003)$ & $.0001(.003)$ & $-.001(.003)$ \\
\hline Depression score & $-.02(.02)$ & $-.02(.02)$ & $-.02(.02)$ \\
\hline Anxiety score & $.005(.006)$ & $.005(.006)$ & $.008(.006)$ \\
\hline Epworth score & $.001(.004)$ & $.001(.004)$ & $.001(.004)$ \\
\hline Session \#3 & $-.04(.01)^{* * *}$ & $-.04(.01)^{* * *}$ & $-.03(.01)^{* * *}$ \\
\hline Circadian mismatched $(=1)$ & $.0004(.02)$ & $-.004(.02)$ & $-.01(.03)$ \\
\hline Nightly sleep (min) & $.0002(.0001)^{*}$ & - & - \\
\hline Personal SD (min/night) & - & $\begin{array}{l}-.0002 \\
(.0001)^{* *}\end{array}$ & $-.0003(.0001)^{* * *}$ \\
\hline Wald chi-squared test (10) & 12.84 & 15.60 & $18.24^{* *}$ \\
\hline
\end{tabular}

Notes: ${ }^{*},{ }^{* *},{ }^{* * *}$ indicate significance at the $.10, .05$, and .01 levels, respectively, for the 2-tailed test.

Model 3 shows that Personal SD more strongly impacts trustworthiness when a strong initial trust signal is received.

Trustworthiness results are shown in Table 6 and Fig. 4. Here, given the strategy-method nature of choice elicitation, the dependent variable is the average percentage passed back by the second-mover over all possible initially trusted amounts. We also include an additional model in Table 6 (far-right column) where we average the percentage pass-back decisions over the subset of amounts where at least half the monetary pie is initially trusted. This evaluates whether sleep restriction impacts trustworthiness differently when the initial signal of trust is most clear. ${ }^{14}$ As can be seen in Table 6 , sleep restriction significantly decreases trustworthiness, and the effect is also robust. Moreover, the effect is largest in magnitude and estimated with the most precision when evaluating trustworthiness in the face of strong initial signals of trust. In other words,

alternative scorings of sleep restriction, and so we choose to focus on the measure Personal SD throughout the main text for displaying the results relative to an easily-understood benchmark of Personal $S D=0$ (where someone is getting exactly the amount of sleep she thinks she needs).

13 That is, subjects were informed prior to making decisions that the risk associated with the first mover decision in this game was not a function of another individual's choice in the current experiment group, but rather the amount "passed back" was a draw from a distribution that reflected typical pass back amounts from previous experiments. Previous research with social decision tasks has highlighted that behavior with a real human counterpart may differ from behavior when matched with a computerized or algorithmic counterpart (e.g., Kosfeld et al., 2005; Krajbich et al., 2009).

${ }^{14}$ In this case, the strength of the initial trust signal is also significant because more than half the pie initially trusted implies the first-mover is exposing herself to being exploited by the trustee. 


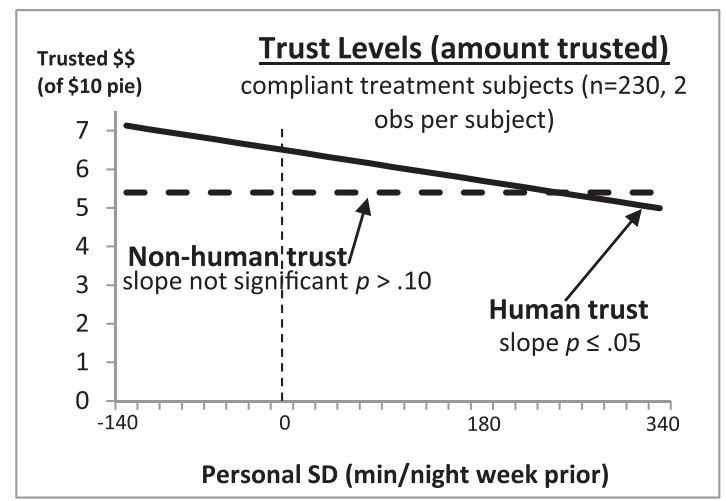

Fig. 3. Predicted trust levels.

Notes: Forecast derived from Table 5 with levels of all statistically insignificant variables set to zero. This figure shows range of values of Personal SD observed in sample of compliant treatment subjects. Results show that when subjects are more chronically sleep restricted, they trust less of the $\$ 10$ pie. Non-human trust line shows predicted trust when 2nd-mover is known to be an automatic pass-back algorithm as opposed to another subject. Trusted amounts in non-human trust are not significantly affected by Personal SD.

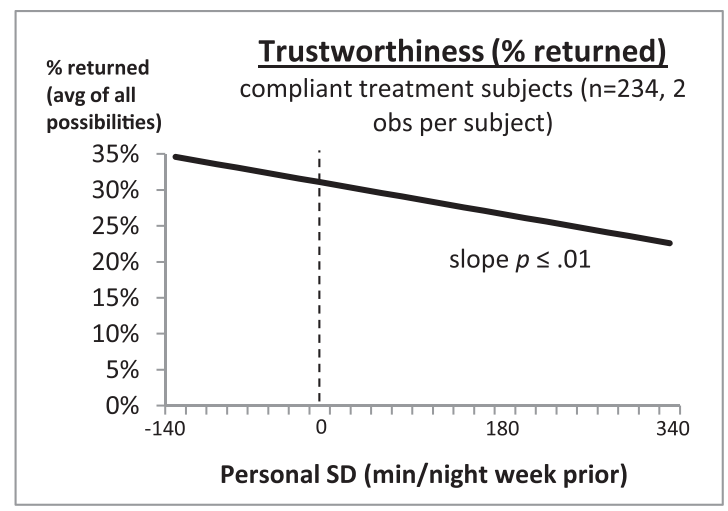

Fig. 4. Predicted trustworthiness.

Notes: Forecast derived from Table 6 with levels of all statistically insignificant variables set to zero. This figure shows range of values of Personal SD observed in sample of compliant treatment subjects. Results show that when subjects are more chronically sleep restricted, they are less trustworthy. (i.e., average \% of pie returned is lower).

sleep restriction harms trustworthiness more when the truster is most vulnerable, which should be concerning. Fig. 4 shows that the effect size of the result in Table 6, column 2, is somewhat smaller than the Dictator or Trust effects of sleep restriction. Nevertheless, it is a robust result that indicates an approximate $7 \%$ reduction in trustworthiness over a baseline rate of $33 \%$ trustworthiness in response to an additional $2 \mathrm{~h}$ of chronic sleep restriction. ${ }^{15}$

Given the Trust game results, one might argue that the SR effect of reduced trust is due to an accurate anticipation of reduced trustworthiness. Our data cannot directly test this, but we reject this interpretation for two reasons: First, the argument is not consistent with the Ultimatum results-there is at least some marginal evidence that SR increases first-mover greed (model 2 in Table 3; Table 7 discussed below), and yet there is no predicted change in MAOs. Second, this interpretation requires anticipation skills that have neural correlates in brain regions harmed by sleep loss (Coricelli and Nagel, 2009). Dickinson and McElroy (2012) also showed direct behavioral evidence that decisions requiring such anticipatory skills suffer from adverse sleep states. ${ }^{16}$

\footnotetext{
15 Here, the baseline trustworthiness of 33\% is the approximate level of predicted trustworthiness for somewhat who is not sleep restricted (Personal $S D=0$ ), and it also represents the Rate-of-Return breakeven level of trustworthiness for first-movers to recuperate their initial "investment" given the tripled-investment rule.

16 Results in Tables 3-6, as well as Appendix Table S2, are also robust to the exclusion of extreme sleeper subjects who were personally sleep deprived $>2.5 \mathrm{~h}$ (SR condition) or $<1 / 2 \mathrm{~h}$ (WR condition). Standard errors increase slightly, though key results are still statistically significant at $p<.10$ or better. The magnitude of the estimated effects from removing the more extreme sleepers, not surprisingly, is slightly reduced.
} 
Table 7

Analysis of sleep restriction and circadian mismatch interaction effects.

\begin{tabular}{|c|c|c|c|c|c|c|}
\hline $\begin{array}{l}\text { Random effects GLS re } \\
\text { Standard errors cluster }\end{array}$ & $\begin{array}{l}\text { ssions } \\
\text { on subject i }\end{array}$ & Ult \$ MAO & $\begin{array}{l}\text { Dictator } \$ \$ \\
\text { Offer }\end{array}$ & $\begin{array}{l}\text { ect) } \\
\text { Trust } \\
\text { person } \$ \$ \\
\text { Offer }\end{array}$ & $\begin{array}{l}\text { Trust } \\
\text { Distribution } \\
\$ \$ \text { Offer }\end{array}$ & $\begin{array}{l}\text { Trust- } \\
\text { worthiness } \\
\% \text { passback }\end{array}$ \\
\hline Constant & $5.26(.54)^{* * *}$ & $4.65(.95)^{* * *}$ & $4.03(1.06)^{* * *}$ & $6.54(1.20)^{* * *}$ & $5.70(1.30)^{* * *}$ & $.31(.08)^{* * *}$ \\
\hline Female $(=1)$ & $-.27(.18)$ & $-.62(.32)^{* *}$ & $.06(.34)$ & $-.24(.39)$ & $-.43(.42)$ & $-.003(.03)$ \\
\hline Age & $-.02(.02)$ & $-.08(.04)^{* *}$ & $-.02(.04)$ & $-.01(.04)$ & $.01(.05)$ & $.0002(.003)$ \\
\hline Depression score & $.11(.12)$ & $.02(.21)$ & $-.18(.23)$ & $-.23(.26)$ & $-.35(.28)$ & $-.02(.02)$ \\
\hline Anxiety score & $-.02(.03)$ & $.06(.07)$ & $.10(.08)$ & $.09(.09)$ & $.09(.09)$ & $.005(.006)$ \\
\hline Epworth score & $-.01(.03)$ & $-.03(.04)$ & $.06(.05)$ & $.01(.06)$ & $-.03(.06)$ & $.001(.004)$ \\
\hline Session \#3 & $-.19(.09)^{* *}$ & $-.59(.13)^{* * *}$ & $-.72(.20)^{* * *}$ & $-.63(.25)^{* *}$ & $-.03(.25)$ & $-.04(.01)^{* * *}$ \\
\hline Morning session $(=1)$ & $.11(.18)$ & $.48(.30)$ & $.30(.33)$ & $.27(.38)$ & $-.39(.41)$ & $.003(.02)$ \\
\hline Morningness score & $.01(.02)$ & $.02(.03)$ & $-.02(.03)$ & $-.06(.04)$ & $-.03(.04)$ & $-.002(.002)$ \\
\hline $\operatorname{MM}(=1)$ & $.06(.22)$ & $.19(.37)$ & $-.43(.45)$ & $-.27(.53)$ & $-.13(.55)$ & $.0001(.03)$ \\
\hline Personal SD & $-.001(.001)$ & $.0004(.001)$ & $-.006(.002)^{* * *}$ & $-.005(.003)^{*}$ & $-.005(.003)^{* *}$ & $-.0002(.0001)^{*}$ \\
\hline Personal SD*MM & $-.001(.001)$ & $-.001(.002)$ & $.001(.003)$ & $.001(.004)$ & $.006(.004)$ & $-.00004(.0002)$ \\
\hline $\mathrm{N}$ & 234 & 236 & 237 & 230 & 236 & 234 \\
\hline Wald $X^{2}(11)$ & 12.50 & $31.75^{* * *}$ & $29.35^{* * *}$ & 16.03 & 10.96 & 15.58 \\
\hline
\end{tabular}

Notes: ${ }^{*},{ }^{*},{ }^{* * *}$ indicate significance at the $.10, .05$, and .01 levels, respectively, for the 2-tailed test

Estimation of the Trustworthiness model using the alternative dependent variable of average returned amount when $50 \%$ or more of pie is trusted shows that the Personal SD reduced trustworthiness when high levels of trust are at stake $(\beta=-.0003, p<.05)$, and there is no significant effect of MM or the Personal $S D^{*} M M$ interaction.

Table 8

Karolinska sleepiness score (KSS) by Condition.

\begin{tabular}{llll}
\hline & \multicolumn{3}{c}{$\begin{array}{l}\text { Robust st } \\
\text { errors } \\
\text { (clustered }\end{array}$} \\
Treatment combination & KSS mean & $\begin{array}{l}\text { on subj) } \\
{[95 \% \mathrm{CI}]}\end{array}$ \\
\hline $\mathrm{SR}=0$ and $\mathrm{MM}=0$ & 3.934 & .173 & {$[3.591,4.278]$} \\
$\mathrm{SR}=1$ and $\mathrm{MM}=0$ & 6.621 & .193 & {$[6.234,7.002]$} \\
$\mathrm{SR}=0$ and $\mathrm{MM}=1$ & 5.018 & .243 & {$[4.535,5.500]$} \\
$\mathrm{SR}=1$ and $\mathrm{MM}=1$ & 6.473 & .218 & {$[6.041,6.906]$} \\
\hline
\end{tabular}

Note: self-reported sleepiness is based on average KSS from each decision session (measures taken at beginning and end of the session).

\subsection{Sleep and circadian mismatch interaction analysis}

Van Dongen and Dinges (2003) and Burke et al. (2015) suggest that sleep and circadian misalignment may have interactive effects, and other behavioral research has suggested that adverse sleep states may have cumulative effects (Dickinson and McElroy, 2010). We explore the possibility of interaction effects in Table 7. Here, we show results of estimations that include an interaction variable between circadian mismatch $(M M=1)$ and the Personal SD variable used previously in the Tables 3-6 specifications. Consistent with these earlier estimations, significant adverse sleep state effects (on Dictator offers, trust, and trustworthiness decisions) are linked to Personal SD and not circadian mismatch. One difference with earlier estimations is that we find subjects with higher Personal SD pass less money in the trust game even when the second mover is a pass-back algorithm. However, this finding should not be considered robust given its absence from other specifications we estimated. Perhaps surprisingly, Table 7 results show no significant interaction effect on Personal $S D^{*} M M$. Thus, our data do not show evidence of a magnified behavioral effect in subjects who are both sleep restricted and circadian mismatched. ${ }^{17}$

Relatedly, Table 8 shows the mean values and confidence intervals of self-reported sleepiness by treatment. Table 8 shows that, while both SR and MM significantly increase sleepiness, it is also clear that the magnitude of the SR effect on sleepiness is larger. The insignificant behavioral effects of MM we report may therefore be attributed to the fact that MM does not increase sleepiness as much as SR in our design, which may reflect the more cumulative effect of nightly chronic partial sleep restriction over the course of an entire week. Additionally, one can see from Table 8 that self-reported sleepiness for someone in both SR and MM states is not significantly higher than for someone only in the SR state. While it is unclear why

\footnotetext{
17 Full marginal effects of a change in MM and Personal SD (PSD) on each treatment variable are given, in general, by $\left.\beta_{\mathrm{MM}}+\beta_{(\mathrm{PSD} * \mathrm{MM})}\right)^{\mathrm{PSD}}$ and $\beta_{\mathrm{PSD}}+\beta_{(\mathrm{PSD} * \mathrm{MM})}{ }^{*} \mathrm{MM}$, respectively (evaluated at the mean levels of the treatment variables). However, the coefficients on the interaction term, $\beta_{\text {(PSD* }}{ }^{*}$, is statistically insignificant in every instance. Our focus is therefore on the main effect of each treatment variable on our behavioral outcomes measures.
} 
Table 9

Instrumental variables estimation to examine sleepiness mediating effects. First-stage estimation of determinants of self-reported sleepiness.

$1^{\text {st }}$-stage regression (errors clustered on subject)

KSleepy estimated using following instruments: Demographic/Session variables: Session\#3, Female, Age,

Anxiety Score, Depression Score, Morning Session, Epworth Score. rMEQ score, Personal SD, Mismatch

\begin{tabular}{ll}
\hline & Predictors of KSleepy: Coefficient (st error) \\
Demographic/Session Indicators & Sleep Related Variables \\
\hline Session\#3: $-.365(.225)$ & Epworth Score: $.073(.035)^{* *}$ \\
Female: $.877(.245)^{* * *}$ & rMEQ score: $.008(.022)$ \\
Age: $.048(.021)^{* *}$ & Personal SD: $.010(.001)^{* * *}$ \\
Anxiety Score: $-.040(.047)$ & Mismatch: $.865(.212)^{* * *}$ \\
Depression Score: $-.282(.155)^{*}$ & \\
Morning Session: $-.649(.208)^{* * *}$ & Model $F$ - test $(10,226)=10.86^{* * *}$ \\
\hline
\end{tabular}

Notes: ${ }^{*}, * * * * *$ indicate significance at $.10, .05$, and .01 levels, respectively.

Experimental treatment variables Personal $S D$ and Mismatch both are estimated to significantly increase self-reported sleepiness $(p<.01)$. Minor differences in number of observations across tasks (e.g., small number of blank responses) are noted in 2nd-stage regression $\mathrm{N}$ below. This implies minor differences in estimated coefficients and standard errors for the 1st-stage regression equations. Shown above are the 1st-stage results of the Dictator game model, which are similar in sign and significance to all other 1-stage equations.

self-reported sleepiness is not higher when one is both SR and MM, the lack of interaction effects in the Table 7 analysis is sensible in light of this finding. ${ }^{18}$

\subsection{Sleepiness as a mediating variable}

Because our manipulations were intended to increase sleepiness, we also evaluate whether sleepiness is the primary mediator of these results (Tables 9 and 10). For the two-step estimation we first regress the Karolinska sleepiness scores (KSS) on demographics, session, and sleep variables (Table 9). As noted earlier, both SR and circadian mismatch significantly predict higher KSS $(p<.01)$, which is seen in the first stage estimation results. This provides validation for our treatment manipulations and identifies the variation in KSS due to subject-specific factors as well. ${ }^{19}$

Step two used the predicted KSS values as a covariate (instrument) in the main decision outcome estimations. Table 10 estimates indicate that higher levels of the KSS-instrument predict lower levels of ultimatum proposals, dictator altruism, trust, and trustworthiness. These estimates indicate that our experimental treatments alter behavioral outcomes through their impact on sleepiness. They also highlight that circadian mismatch, while not estimated to directly impact behavioral outcomes, is estimated to indirectly affect social behavior via its impact on sleepiness. In conjunction with our other analysis represented in Tables 3-6, these results describe a common theme whereby prosocial decisions decline due to sleepiness that is symptomatic of an adverse sleep or circadian state.

\section{Discussion}

We examined the impact of mild but chronic sleep restriction and suboptimal circadian timing on decisions in simple social interactions. The sleep levels studied and the times-of-day utilized are highly relevant to what a large segment of the population experiences in everyday life. Because we utilized an ecologically valid setting, and compensatory strategies to combat the sleepiness were not prohibited, our results can be viewed as conservative estimates of the impact of common sleep states in the simple games we examined.

The simple social decision tasks we used are well-known and they form the building blocks of more complex interactive environments where social behaviors loom large. We found robust results consistent with the hypothesis that commonly experienced adverse sleep states reduce prosocial behaviors. Our results showing reduced dictator giving are the most precisely estimated, although trust and trustworthiness are also found to decrease significantly in most specifications. We hypothesized that adverse sleep states would likely reduce prosocial behaviors due to their tendency to reduce deliberative thought. While we do not have direct evidence that our sleep and circadian manipulations impacted prefrontal activation,

\footnotetext{
18 It is possible that subjects in a condition of both SR and MM took countermeasures to counteract sleepiness (other than naps, which would contribute to noncompliance). However, the one item on which we collected countermeasure data during the decision session-whether caffeine or sugar was consumed in the $3 \mathrm{~h}$ prior to the lab session-did not show significant differences across individual or combined sleep conditions (see Appendix Table S1). These data are, however, not precise in terms of standardized size and caffeine/sugar content-we used simple count data on self-reported caffeine/sugar consumption prior to the session as a $0 / 1$ variable).

19 We also note that the impact of gender is implicit in these results. First-stage estimates show that female subjects are significantly sleepier, controlling for other demographics and sleep variables.
} 
Table 10

Instrumental variables estimations to examine sleepiness mediating effects. Second-stage estimations of behavioral outcomes using instrumented values of self-reported sleepiness.

\begin{tabular}{|c|c|c|c|c|c|c|}
\hline \multicolumn{7}{|c|}{$\begin{array}{l}2^{\text {nd }} \text {-stage regression: KSleepy instrumented from } 1 \text { st-stage regression } \\
\text { Standard errors clustered on subject ( } 2 \text { observations per subject) }\end{array}$} \\
\hline Variable & Ultimatum $\$ \$$ Offer & Ultimatum $\$ \$ M A O$ & Dictator $\$ \$$ Offer & Trust person $\$ \$$ Offer & Trust algorithm $\$ \$$ Offer & Trust-worthiness \% passback \\
\hline KSleepy(instr) & $-.17(.08)^{* *}$ & $-.04(13)$ & $-.46(.16)^{* * *}$ & $-.35(.16)^{* *}$ & $--17(.17)$ & $-.02(.01)^{* *}$ \\
\hline Session \#3 & $-.25(.10)^{* *}$ & $-.60(.14)^{* * *}$ & $-.88(.22)^{* * * *}$ & $-.79(.27)^{* * *}$ & $-.10(.25)$ & $-.04(.02)^{* * *}$ \\
\hline Female & $-.13(.15)$ & $-.59(.31)^{*}$ & $.42(.37)$ & $-.002(.04)$ & $-.41(.45)$ & $.01(.03)$ \\
\hline age & $-.01(.02)$ & $-.06(.04)$ & $-.02(.04)$ & $-.02(.04)$ & $-.005(.04)$ & $.0001(.002)$ \\
\hline Depression score & $.06(.09)$ & .002 (.19) & $-.30(.22)$ & $-.27(.27)$ & $-.34(.28)$ & $-.02(.02)$ \\
\hline Anxiety score & $-.03(.03)$ & $.05(.07)$ & $.12(.07)$ & $.10(.07)$ & $.09(.07)$ & $.005(.005)$ \\
\hline Constant & $5.91(.71)^{* * *}$ & $4.83(1.19)^{* * *}$ & $5.93(1.31)^{* * * *}$ & $7.44(1.22)^{* *}$ & $5.75(1.27)^{* * *}$ & $.38(.09)^{* * * *}$ \\
\hline $\mathrm{N}$ & 234 & 236 & 237 & 230 & 236 & 234 \\
\hline Wald $X^{2}$ & 10.42 & $30.97 * * *$ & $25.45^{* * *}$ & $12.19^{*}$ & 5.89 & $11.91 *$ \\
\hline
\end{tabular}

Notes: ${ }^{*},{ }^{* *},{ }^{* * *}$ indicate significance at the $.10, .05$, and .01 levels, respectively, for the 2-tailed test.

The same 2SLS model run using the alternative Trustworthiness variable (i.e., average percentage passed back on all possible first-mover trusted amounts of at least half the pie), shows similar results to the Trustworthiness model above, except that the magnitude of the instrumented KSleepy variable is slightly larger in magnitude (-.023 vs -.019 ) and estimated a bit more precisely ( $p=.03 \mathrm{vs} p=.038$ ). This is consistent with what we report in the text that the impact of sleep restriction (here, via the mediating variable, KSleepy) to reduce trustworthiness is somewhat magnified for those decisions involving a more sizeable and clear signal of trust by the first-mover 
a body of literature in sleep science clearly links more extreme adverse sleep states (e.g., total sleep deprivation) to disproportionate reductions in PFC activation, as well as altered emotional response region activation. For example, sleepy subjects may experience increased fear of betrayal as a mechanism that results in reduced trust, as was proposed in an earlier study (Anderson and Dickinson, 2010). While suggestive, we also note that our sleep restriction manipulation produced the same general behavioral effects as others have found in patients with vmPFC damage (Krajbich et al., 2009). Future research may wish to document the impact of these more mild sleep/circadian manipulations on neural activation patterns in order to establish direct evidence for the mechanism that drives this behavioral result.

Our findings imply that commonly experienced adverse sleep states reduce prosocial behaviors and lead to unrealized gains in simple social interactions. A reduction in an individual's social capital has multiplier effects, and so any factor that can be shown to reduce prosocial behavior is noteworthy. One of the main criticisms of recent work identifying the decline in social capital in America (Putman, 1995) is the fact that many of the arguments are based on correlational data (see critique in Sobel, 2002). While we do not claim to have uncovered the hidden source of decreased social capital during recent decades, we do stand on firmer ground in claiming at least one micro-level causal determinant of reduced prosocial behavior in these simple environments.

\subsection{Conclusions}

Given the prevalence of social interactions in everyday life, it should be concerning to identify that sleep restriction adversely impacts prosocial behavior. This research helps show that a common trend in current adult sleep habits may not just affect decision outcomes, but it also may undermine the potential to generate value in interpersonal interactions and possibly reduce social capital multiplier effects. Such social "inefficiency" costs of sleepiness are not fully appreciated and largely ignored. Future research should seek to evaluate whether these effects are also robust to repeated, longer-term, or non-anonymous interactions. In the meantime, it seems like a piece of timely advice for encouraging prosocial behavior would be to tell everyone to "just sleep on it".

\section{Acknowledgments}

This work was supported by National Science Foundation grant BCS-1229067. We thank Sean P.A. Drummond for assistance designing the sleep protocol, T. Perry, A. Beckett, L. Morris, R. McClelland, and E. Wilson for help with sessions, and $\mathrm{N}$. Stroh and S. Rule for assistance with decision tasks and sleep data preparation and scoring. Comments from seminar participants at numerous universities are gratefully acknowledged. The authors also thank Mike McKee for helpful comments. Data are available from Dickinson on request.

\section{Supplementary material}

Supplementary material associated with this article can be found, in the online version, at 10.1016/j.euroecorev.2017.05. 002.

\section{References}

Achtziger, A., Alós-Ferrer, C., Wagner, A.K., 2016. The impact of self-control depletion on social preferences in the ultimatum game. J. Econ. Psychol. 53 (Apr), 1-16.

Adan, A., Admiral, H., 1991. Horne and Ostberg morningness-eveningness questionnaire: a reduced scale. Pers. Indiv. Dif. 12 (3), $241-253$.

Ainsworth, S.E., Baumeister, R.F., Ariely, D., Vohs, K.D., 2014. Ego depletion decreases trust in economic decision making. J. Exp. Social Psychol. 54 (Sept 30 ), 40-49.

Åkerstedt, T., Gillberg, M., 1990. Subjective and objective sleepiness in the active individual. Int. J. Neurosci. 52 (1-2), 29-37.

Alhola, P., Polo-Kantola, P., 2007. Sleep deprivation: impact on cognitive performance. Neuropsychiatr. Dis. Treat. 3 (5), 553-567.

Almklov, E.L., Drummond, S.P., Orff, H., Alhassoon, O.M., 2015. The effects of sleep deprivation on brain functioning in older adults. Behav. Sleep Med. 13 (4), 324-345.

Anderson, C., Dickinson, D.L., 2010. Bargaining and trust: the effects of $36 \mathrm{hr}$ sleep deprivation on socially interactive decisions. J. Sleep Res. 19 (1-Part-1), 54-63.

Benenson, J.F., Pascoe, J., Radmore, N., 2007. Children's altruistic behavior in the dictator game. Evol. Hum. Behav. 28 (3), 168-175.

Berg, J.E., Dickhaut, J.W., McCabe, K.A., 1995. Trust, reciprocity, and social history. Game. Econ. Behav. 10 (1), 122-142.

Bodenhausen, G.V., 1990. Stereotypes as judgmental heuristics: evidence of circadian variations in discrimination. Psychol. Sci. 1 (5), 319-322.

Burke, T.M., Scheer, F.A.J.L., Ronda, J.M., Czeisler, C.A., Wright, K.P., 2015. Sleep inertia, sleep homeostatic and circadian influences on higher-order cognitive functions. J. Sleep Res. 24 (4), 364-371.

Chee, M.W., Chuah, L.Y., 2008. Functional neuroimaging insights into how sleep and sleep deprivation affect memory and cognition. Curr. Opin. Neurol. 21 (4), 417-423.

Chelminski, I., Petros, T.V., Plaud, J.J., Ferraro, F.R., 2000. Psychometric properties of the reduced Horne and Östberg questionnaire. Pers. Indiv. Dif. 29 (3), $469-478$.

Coricelli, G., Nagel, R., 2009. Neural correlates of depth of strategic reasoning in medial prefrontal cortex. Proc. Natl. Acad. Sci. USA 106 (23), 9163-9168.

Dickinson, D.L., Drummond, S.P.A., McElroy, T., 2017. The viability of an ecologically valid chronic sleep restriction and circadian timing protocol: an examination of sample attrition, compliance, and effectiveness at impacting sleepiness and mood. PLoS ONE 12 (3), e0174367.

Dickinson, D.L., McElroy, T., 2010. Rationality around the clock: sleep and time-of-day effects on guessing game responses. Econ. Lett. 108 (2), 245-248.

Dickinson, D.L., McElroy, T., 2012. Circadian effects on strategic reasoning. Exp. Econ. 15 (3), 444-459.

Drummond, S.P.A., Brown, G.G., Gillin, J.C., Stricker, J.L., Wong, E.C., Buxton, R.B., 2000. Altered brain response to verbal learning following sleep deprivation. Nature 403 (6770), 655-657. 
Drummond, S.P., Brown, G.G., Salamat, J.S., Gillin, J.C., 2004. Increasing task difficulty facilitates the cerebral compensatory response to total sleep deprivation. Sleep 27 (3), 445-452.

Falk, A., Meier, S., Zehnder, C., 2013. Do lab experiments misrepresent social preferences? The case of self-selected student samples. J. Eur. Econ. Assoc. 11 (4), 839-852

Fehr, E., Bernhard, H., Rockenbach, B., 2008. Egalitarianism in young children. Nature 454 (7208), 1079-1083.

Fehr, E., Camerer, C.F., 2007. Social neuroeconomics: the neural circuitry of social preferences. Trends Cogn. Sci. 11 (10), 419-427.

Fehr, E., Glätzle-Rützler, D., Sutter, M., 2013. The development of egalitarianism, altruism, spite and parochialism in childhood and adolescence. Eur. Econ. Rev. 64 (Nov), 369-383.

Fehr, E., Schmidt, K.M., 1999. A theory of fairness, competition, and cooperation. Q. J. Econ. (Aug) 817-868.

Ferrara, M., Bottasso, A., Tempesta, D., Carrieri, M., De Gennaro, L., Ponti, G., 2015. Gender differences in sleep deprivation effects on risk and inequality aversion: evidence from an economic experiment. PLoS ONE 10 (3), e0120029.

Forsythe, R., Horowitz, J.L., Savin, N.E., Sefton, M., 1994. Fairness in simple bargaining games. Game Econ. Behav. 6 (3), 347-369.

Glaeser, E.L., Laibson, D., Sacerdote, B., 2002. An economic approach to social capital. Econ. J. 112 (483), F437-F458.

Güth, W., Schmittberger, R., Schwarze, B., 1982. An experimental analysis of ultimatum bargaining. J. Econ. Behav. Organ. 3 (4), 367-388 3. Schoenborn CA, Adams PF (2010) Health behaviors of adults: United States, 2005-2007. National Center for Health Statistics. Vital Health Stat, series 10, Data from the National Health Survey 245: 1-132.

Harrison, Y., Horne, J.A., 2000. The impact of sleep deprivation on decision making: a review. J. Exp. Psychol.: Appl. 6 (3), 236.

Horne, J.A., Östberg, O., 1976. A self-assessment questionnaire to determine morningness-eveningness in human circadian rhythms. Int. J. Chronobiol. 4 (2), 97-110.

Horne, J.A., 1993. Human sleep, sleep loss and behavior. Implications for the prefrontal cortex and psychiatric behavior. Br. J. Psychiatr. 162, 413-419.

Inzlicht, M., Schmeichel, B.J., 2012. What is ego depletion? Toward a mechanistic revision of the resource model of self-control. Perspect. Psychol. Sci. 7 (5), 450-463.

Kaida, K., Takahashi, M., Åkerstedt, T., Nakata, A., Otsuka, Y., Haratani, T., Fukasawa, K., 2006. Validation of the Karolinska sleepiness scale against performance and EEG variables. Clin. Neurophysiol. 117 (7), 1574-1581.

Killgore, W.D.S., Balkin, T.J., Wesensten, N.J., 2006. Impaired decision making following 49 h of sleep deprivation. J. Sleep Res. 15 (1), 7-13.

Killgore, W.D.S., Grugle, N.L., Balkin, T.J., 2012. Gambling when sleep deprived: don't bet on stimulants. Chronobiol. Int. 29 (1), 43-54.

Knack, S., Keefer, P., 2007. Does social capital have an economic payoff? A cross-country investigation. Q. J. Econ. 112 (4), $1251-1288$.

Kosfeld, M., Heinrichs, M., Zak, P.J., Fischbacher, U., Fehr, E., 2005. Oxytocin increases trust in humans. Nature 435 (7042), 673-676.

Krajbich, I., Adolphs, R., Tranel, D., Denburg, N.L., Camerer, C.F., 2009. Economic games quantify diminished sense of guilt in patients with damage to the prefrontal cortex. J. Neurosci. 29 (7), 2188-2192.

Krajbich, I., Björn, B., Hare, T., Fehr, E., 2015. Rethinking fast and slow based on a critique of reaction-time reverse inference. Nat. Commun. 6 (Jul 2), 7455.

Kruglanski, A.W., Pierro, A., 2008. Night and day, you are the one on circadian mismatches and the transference effect in social perception. Psychol. Sci. 19 (3), 296-301.

LaPorta, R., Lopez-de-Salanes, F., Shleifer, A., Vishny, R., 1997. Trust in large organisations. Am. Econ. Rev. Papers Proc. 87, 333-338.

Levitt, S.D., List, J.A., 2007. What do laboratory experiments measuring social preferences reveal about the real world? J. Econ. Perspect. 21 (2), $153-174$.

McCabe, K., Houser, D., Ryan, L., Smith, V., Trouard, T., 2001. A functional imaging study of cooperation in two-person reciprocal exchange. Proc. Natl. Acad. Sci. USA 98 (20), 11832-11835.

McElroy, T., Dickinson, D.L., 2010. Thoughtful days and valenced nights: how much will you think about the problem? Judg. Decis. Making 5 (7), 516-523.

Muzur, A., Pace-Schott, E.F., Hobson, J.A., 2002. The prefrontal cortex in sleep. Trends Cogn. Sci. 6 (11), 475-481.

Putnam, R.D., 1993. Making Democracy Work: Civic Traditions in Modern Italy. Princeton University Press, Princeton.

Putnam, R.D., 1995. Bowling alone: America's declining social capital. J. Democracy. 6 (1), 65-78.

Rand, D.G., Brescoll, V.L., Everett, J.A.C., Capraro, C., Barcelo, H., 2016. Social heuristics and social roles: intuition favors altruism for women but not for men. J. Exp. Psychol. Gen. 145 (4), 389-396.

Rand, D.G., Greene, J.D., Nowak, M.A., 2012. Spontaneous giving and calculated greed. Nature 489 (7416), 427-430.

Rand, D.G., et al., 2014. Social heuristics shape intuitive cooperation. Nat. Commun. 5, 3677. doi:10.1038/ncomms4677.

Rilling, J.K., Sanfey, A.G., 2011. The neuroscience of social decision-making. Annu. Rev. Psychol. 62 (Jan 10), 23-48.

Sanfey, A.G., et al., 2003. The neural basis of economic decision-making in the ultimatum game. Science 300 (5626), 1755-1758.

Schoenborn, C.A., Adams, P.F., 2010. Health behaviors of adults: United States, 2005-2007. National Center for Health Statistics. Vital Health Stat, series 10. Data Natl. Health Surv. 245, 1-132.

Sobel, J., 2002. Can we trust social capital? J. Econ. Lit. 40 (1), 139-154.

Tinghög, G., Andersson, D., Bonn, C., Böttiger, H., Josephson, C., et al., 2013. Intuition and cooperation reconsidered. Nature 498 (7452), E1-E2.

Van Dongen, H.P.A., Dinges, D.F., 2003. Investigating the interaction between the homeostatic and circadian processes of sleep-wake regulation for the prediction of waking neurobehavioural performance. J. Sleep Res. 12 (3), 181-187.

Venkatraman, V., Chuah, L., Huettel, S.A., Chee, M.W., 2007. Sleep deprivation elevates expectation of gains and attenuates response to losses following risky decisions. Sleep 30, 603-609.

Venkatraman, V., Huettel, S.A., Chuah, L.Y., Payne, J.W., Chee, M.W., 2011. Sleep deprivation biases the neural mechanisms underlying economic preferences. J. Neurosci. 31 (10), 3712-3718.

Venkatraman, V., Payne, J.W., Bettman, J.R., Luce, M.F., Huettel, S.A., 2009. Separate neural mechanisms underlie choices and strategic preferences in risky decision making. Neuron $62(4), 593-602$.

Verkoeijen, P.P.J.L., Bouwmeester, S., 2014. Does intuition cause cooperation? PLOS ONE. 9 (5), e96654

Vohs, K.D., Glass, B.D., Maddox, W.T., Markman, A.B., 2010. Ego depletion is not just fatigue: evidence from a total sleep deprivation experiment. Social Psychol. Personality Sci. 2 (2), 166-173.

Yoo, S.S., Guhar, N., Hu, P., Jolesz, F.A., Walker, M.P., 2007. The human emotional brain without sleep-a prefrontal amygdale disconnect. Curr. Biol. 17 (20), $877-878$ 